\title{
Anisotropy and state mixing in the interactions between Rydberg states
}

\author{
Benoît Vermersch ${ }^{1,2, \text { a }}$ \\ 1 Institute for Quantum Optics and Quantum Information of the Austrian Academy of \\ Sciences, A-6020 Innsbruck, Austria \\ 2 Institute for Theoretical Physics, University of Innsbruck, A-6020 Innsbruck, Austria
}

Received 31 December 2015 / Received in final form 12 June 2016

Published online 19 December 2016

\begin{abstract}
We present a guide to calculate the interactions between Rydberg states, and put into evidence the importance of the anisotropic and state-mixing character of these interactions for $p$ and $d$ states. We then summarize recent theoretical results obtained in this context related to the formation of anisotropic Rydberg excitation patterns and the influence of state-mixing on the familiar Rydberg blockade phenomenon.
\end{abstract}

\section{Introduction}

Rydberg states represent highly excited electronic states of atoms. Due to the large orbital radius of the valence electron, such states are long-lived and induce strong interactions between the atoms [1], which make them a very natural platform to observe quantum many-body phases [2], and a very promising candidate for quantum information applications [3]. With recent experimental developments, it became possible to arrange cold atoms in well defined geometries, excite them to a Rydberg level, and obtain single-site resolved measurements, allowing to demonstrate blockade physics $[4,5]$ between two atoms [6,7], and its consequences at the many-body level [8-14].

Recently, due to the possibility to excite Rydberg states from a ground state level, via a single photon transition, Rydberg $p$ states have drawn attention. However, in contrast to the familiar $s_{1 / 2}$ states, the interactions between Rydberg $p$ or $d$ states are anisotropic, i.e. the energy potential depends on the angle between the displacement vector of the atoms and the magnetic field [15]. Moreover, the interactions do not conserve in general the electronic state of the atoms [16,17], such "state-mixing" not being taken into account in the traditional Rydberg blockade picture.

The goal of this article is to review the theoretical tools, which allow to describe the interactions between Rydberg states, either at short distances (dipole-dipole regime) or large distances (van der Waals regime), and show the presence of the anisotropic and state-mixing character of these interactions. First, in Sect. 2, we present a concise guide allowing to calculate Rydberg wave-functions and the corresponding dipoledipole Hamiltonian. In Sect. 3, we obtain the van der Waals Hamiltonian and illustrate the interacting properties of Rydberg $s, p, d, f$ states with various examples.

\footnotetext{
${ }^{a}$ e-mail: benoit.vermersch@uibk.ac.at
} 


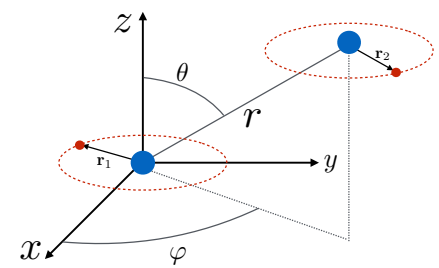

Fig. 1. Interactions between two Rydberg atoms, parametrized in terms of spherical coordinates $(r, \theta, \varphi)$. The blue circles represent the positively charged cores (nucleus and inner electrons) and the red circles represent the two electrons.

Finally, in Sect. 4, we summarize our recent results $[18,19]$ in this context, showing the anisotropic properties of Rydberg gases in optical lattices and the effect of state-mixing on the Rydberg blockade phenomenon.

\section{Dipole-dipole interactions between Rydberg states}

In this section, we show how to calculate the dipole-dipole interactions between two Rydberg atoms. To do so, we write the dipole-dipole Hamiltonian in the fine-structure basis, and present a guide to calculate dipole matrix elements using model potentials [20] and the knowledge of the Rydberg energy levels.

The situation we have in mind is shown in Fig. 1: we consider two Rydberg atoms $i=1,2$, separated by a vector $\mathbf{r}$. Our goal is to describe the Coulomb interaction between these two inhomogeneous charge distributions, in the regime where the distance $r$ between the atoms is much larger than the typical orbital radius $r_{i}$ of a Rydberg electron around the nucleus. In this case, the Coulomb potential can be written using a multipole expansion, the dominant contribution being the dipole-dipole interaction [3]:

$$
H^{(d d)}=\frac{r^{2} \mathbf{d}^{(1)} \cdot \mathbf{d}^{(2)}-3\left(\mathbf{d}^{(1)} \cdot \mathbf{r}\right)\left(\mathbf{d}^{(2)} \cdot \mathbf{r}\right)}{r^{5}},
$$

which is written here in atomic units, with the dipole operator $\mathbf{d}^{(i)}=-\hat{\mathbf{r}}_{i}$ associated with the Rydberg electron's position of atom $i$. In order to be able to apply this Hamiltonian for any angular momentum state, we now write this Hamiltonian in spherical coordinates [21] defining the spherical components of the dipole operator $d_{ \pm 1}=\mp 1 / \sqrt{2}\left(d_{x} \pm i d_{y}\right), d_{0}=d_{z}$, where $\mathbf{z}$ is the quantization axis. We obtain:

$$
H^{(d d)}=-\sqrt{\frac{24 \pi}{5}} \frac{1}{r^{3}} \sum_{m_{1}, m_{2}}\left[\begin{array}{cc|c}
1 & 1 & 2 \\
m_{1} & m_{2} & m_{1}+m_{2}
\end{array}\right] Y_{2, m_{1}+m_{2}}^{*}(\theta, \varphi) d_{m_{1}}^{(1)} d_{m_{2}}^{(2)}
$$

Here $(r, \theta, \varphi)$ are the spherical coordinates of the vector connecting the two atoms, with respect to the quantization axis $\mathbf{z}$. The square brackets denote a Clebsch-Gordan coefficient and $Y_{l, m}(\theta, \varphi)$ the spherical harmonics functions. We now explain in detail how this Hamiltonian couples Rydberg states.

\subsection{Calculation of dipole matrix elements in the fine-structure}

In the fine-structure representation, a Rydberg state, denoted $a$, is characterized by the set of quantum numbers $n_{a}, \ell_{a}, j_{a}, s_{a}, m_{a}$ corresponding to a wavefunction $\left|n_{a} X_{a} m_{a}\right\rangle \equiv R_{n_{a} X_{a}}\left(r_{i}\right)\left|X_{a}, m_{a}\right\rangle$, where we use the short-hand notation 
$X_{a}=\left(\ell_{a}, j_{a}, s_{a}\right)$ and consider in all this work a single valence electron $s_{a}=1 / 2$. The decomposition of the wave-function into a radial part $R_{n_{a} X_{a}}\left(r_{i}\right)$ and an angular part $\left|X_{a}, m_{a}\right\rangle$ allows to write the matrix elements of the dipole operator of the atom $i$ between two Rydberg states $a$ and $c$ as:

$$
\left\langle n_{a} X_{a} m_{a}\left|d_{m}^{(i)}\right| n_{c} X_{c} m_{c}\right\rangle=I_{n_{a} X_{a}, n_{c} X_{c}} J_{m, X_{a} m_{a}, X_{b} m_{b}} .
$$

The calculation of the radial part

$$
I_{n_{a} X_{a}, n_{c} X_{c}}=\int \mathrm{R}_{n_{a} X_{a}}\left(r_{i}\right) \mathrm{R}_{n_{c} X_{c}}\left(r_{i}\right) r_{i}^{3} d r_{i}
$$

requires the knowledge of the radial wave-function, whereas the angular part is an analytic function of the quantum numbers [17]:

$$
\begin{aligned}
J_{m, X_{a} m_{a}, X_{c} m_{c}=} & (-1)^{j_{c}+\ell_{a}+1 / 2} \sqrt{\left(2 j_{c}+1\right)\left(2 \ell_{c}+1\right)} \\
& \times\left[\begin{array}{cc|c}
j_{c} & 1 & j_{a} \\
m_{c} & m & m_{a}
\end{array}\right]\left[\begin{array}{cc|c}
\ell_{c} & 1 & \ell_{a} \\
0 & 0 & 0
\end{array}\right]\left\{\begin{array}{ccc}
\ell_{c} & 1 / 2 & j_{c} \\
j_{a} & 1 & \ell_{a}
\end{array}\right\},
\end{aligned}
$$

where the braces in the first line denote a Wigner $6 j$ symbol.

\subsubsection{Radial matrix elements}

The last ingredient required for the evaluation of the matrix elements of the dipoledipole Hamiltonian is the radial wave-function, which enters in the calculation of the radial integral (4). For Rydberg states, the radial wave-function can be calculated from the radial Schrödinger equation

$$
\left[-\frac{1}{2} \nabla_{r_{i}}^{2}+\frac{\ell_{a}\left(\ell_{a}+1\right)}{2 r_{i}^{2}}+V\left(r_{i}\right)\right] R_{n_{a} X_{a}}\left(r_{i}\right)=E_{n_{a} X_{a}} R_{n_{a} X_{a}}\left(r_{i}\right) .
$$

The Rydberg electron is subject to the potential $V\left(r_{i}\right)=V_{\bmod }\left(r_{i}\right)+V_{\text {so }}\left(r_{i}\right)$, where $V_{\text {mod }}\left(r_{i}\right)$ corresponds to a model potential $[1,20]$ describing the interaction between the electron and the nucleus, beyond the Hydrogen approximation, and

$$
V_{\mathrm{so}}\left(r_{i}\right)=\frac{\alpha^{2}}{2 r_{i}^{3}} \mathbf{L} \cdot \mathbf{S}=\frac{\alpha^{2}}{4 r_{i}^{3}}\left[j_{a}\left(j_{a}+1\right)-\ell_{a}\left(\ell_{a}+1\right)-s_{a}\left(s_{a}+1\right)\right]
$$

represents the familiar spin-orbit contribution. Finally, $E_{n_{a} X_{a}}$ represents the energy of the Rydberg state $a$. There are two options to calculate Rydberg radial wavefunctions based on Eq. (6). A first possibility is to numerically solve this equation as an eigenvalue problem, and obtain the set $\left(R_{n_{a} X_{a}}\left(r_{i}\right), E_{n_{a} X_{a}}\right)$ characterizing the Rydberg state. An alternative approach consists in directly integrating the radial equation using the experimental values $[22,23]$ of the Rydberg energies $E_{n_{a} X_{a}}$. Such an integration can be carried out via the Numerov method (see for example [24]).

As an illustration, we show in Fig. 2 the radial wave-function obtained for different Rydberg states. The typical extension of the Rydberg wave-function scales as $n^{2}$ whereas the difference between the different curves, obtained for $\ell_{a}=s, p, d, f$, resembles a phase-shift, which can be associated with the different values of the quantum defect [1]. On the other hand, the effect of the spin-orbit coupling, which also manifests in the value of the quantum defect, is not visible on such curves, the difference between the radial wavefunctions of, for example, $p_{1 / 2}$ and $p_{3 / 2}$ states, could hardly be observed. 
(a)

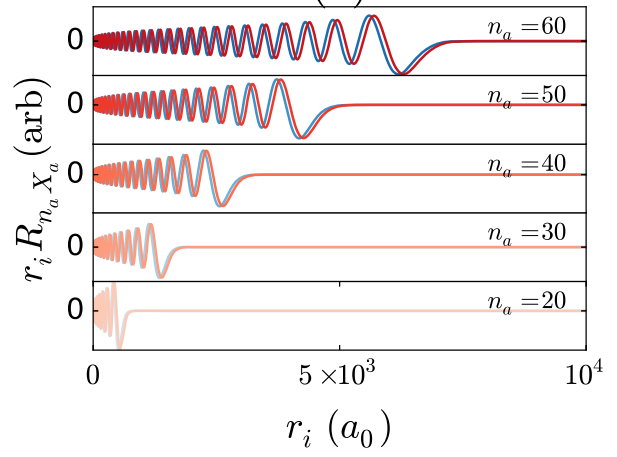

(b)

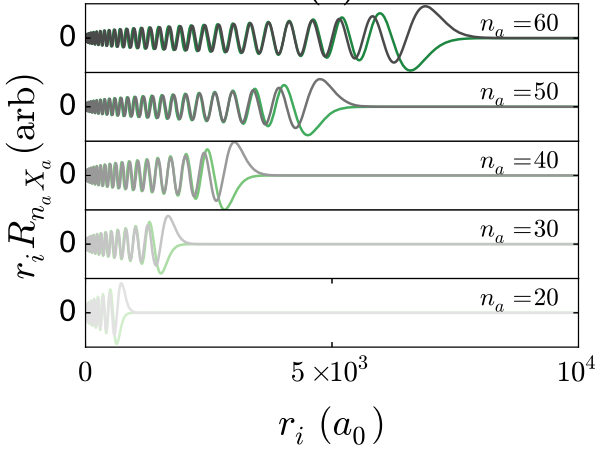

Fig. 2. Radial wave-functions for Rubidium Rydberg atoms, in a $n_{a} s_{1 / 2}, n_{a} p_{1 / 2}$ state [panel (a)], $n_{a} d_{3 / 2}, n_{a} f_{5 / 2}$ [panel (b)], represented as a blue, red, green, grey line, respectively. The distance of the electron $r_{i}$ with respect to the nucleus is expressed in units of the Bohr radius $a_{0}$.

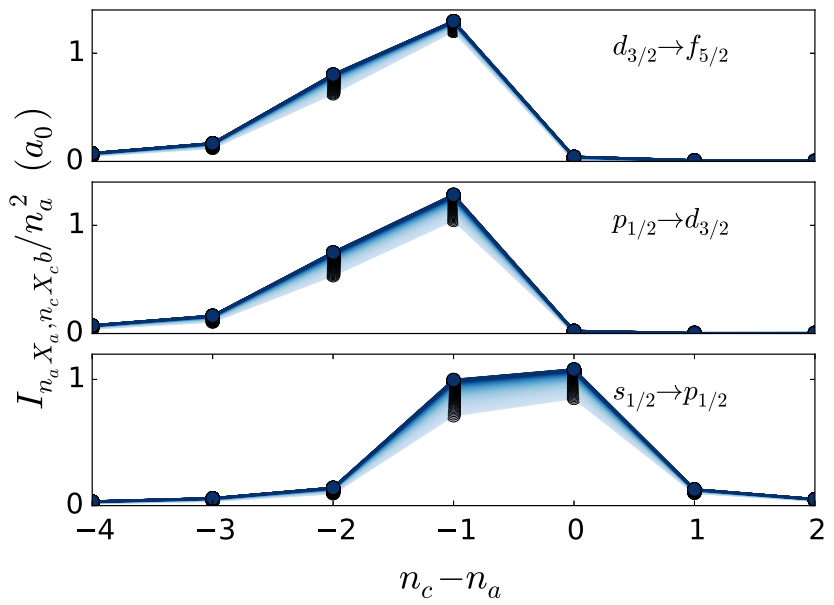

Fig. 3. Rubidium Radial matrix elements $I_{n_{a} X_{a}, n_{c} X_{c}}$ as a function of $n_{c}-n_{a}$ and for different processes $\ell_{a}, j_{a} \rightarrow \ell_{c}, j_{c}$ corresponding to the three panels. The color code indicates the value of $n_{a}$, from 20 (light blue) to 100 (dark blue).

The evaluation of the radial matrix elements $I_{n_{a} X_{a}, n_{c} X_{c}}$ is now straightforward and we present in Fig. 3 the values obtained for different processes $n_{a} X_{a} \rightarrow n_{c} X_{c}$, which are indicated in each panel. One can notice that the values of these integrals only take non-negligible values in a small set of values $n_{c}-n_{a}$ around 0 , corresponding to the regions where the two radial wave-functions $R_{n_{a}, X_{a}}, R_{n_{c}, X_{c}}$ overlap significantly.

\subsection{Dipole-dipole Hamiltonian in fine-structure basis}

We can now write explicitly the coupling between Rydberg atoms by the dipoledipole Hamiltonian Eq. (2). Considering the process where the two-atom state is $\left|n_{c} X_{c} m_{c}, n_{d} X_{d} m_{d}\right\rangle$ (The first atom is in the state $\left|n_{c} X_{c} m_{c}\right\rangle$, whereas the other is in state $\left|n_{d} X_{d} m_{d}\right\rangle$.) are transferred to the state $\left|n_{a} X_{a} m_{a}, n_{b} X_{b} m_{b}\right\rangle$, the decomposition 
of the dipole matrix elements into radial and angular part leads to the following dipole-dipole matrix element:

$$
\begin{aligned}
& \left\langle n_{a} X_{a} m_{a}, n_{b} X_{b} m_{b}\left|H^{(d d)}\right| n_{c} X_{c} m_{c}, n_{d} X_{d} m_{d}\right\rangle \\
& \quad=\frac{C_{3}\left(n_{a} X_{a} n_{b} X_{b}, n_{c} X_{c} n_{d} X_{d}\right)}{r^{3}} \times \mathcal{D}\left(X_{a} m_{a} X_{b} m_{b}, X_{c} m_{c} X_{d} m_{d}\right),
\end{aligned}
$$

where the radial $C_{3}$ coefficient is a simple product of radial integrals

$$
C_{3}\left(n_{a} X_{a} n_{b} X_{b}, n_{c} X_{c} n_{d} X_{d}\right)=I_{n_{a} X_{a}, n_{c} X_{c}} I_{n_{b} X_{b}, n_{d} X_{d}}
$$

and the angular part has an analytic expression.

$$
\begin{aligned}
& \mathcal{D}\left(X_{a} m_{a} X_{b} m_{b}, X_{c} m_{c} X_{d} m_{d}\right)=-\sqrt{\frac{24 \pi}{5}} \sum_{m_{1}, m_{2}}\left[\begin{array}{cc|c}
1 & 1 & 2 \\
m_{1} & m_{2} & m_{1}+m_{2}
\end{array}\right] \\
& \times Y_{2, m_{1}+m_{2}}^{*}(\theta, \varphi) J_{m_{1}, X_{a} m_{a}, X_{c} m_{c}} J_{m_{2}, X_{b} m_{b}, X_{d} m_{d} .}
\end{aligned}
$$

Note that the Wigner symbol and Clebsch-Gordan coefficients appearing in the expression of the angular part of the dipole matrix element Eq. (5) and in Eq. (10) are associated with a set of symmetry rules of the dipole-dipole interactions. (i) The dipole-dipole interaction has an odd symmetry: $\left|\ell_{c}-\ell_{a}\right|=1$ and $\left|\ell_{d}-\ell_{b}\right|=1$, i.e. the dipole-dipole interaction only couples different neighboring orbital quantum numbers. (ii) The magnetic quantum number is not necessarily conserved but varies by at most one unit: $m_{c}-m_{a}=-m_{1}=-1,0,1, m_{d}-m_{b}=-m_{2}=-1,0,1$. Finally, we emphasize that in the case, where the total magnetic quantum number is conserved, $\Delta m=m_{1}+m_{2}=0$, the contribution of the spherical harmonics $Y_{2,0}(\theta, \phi) \propto 1-3 \cos ^{2} \theta$ corresponds to the generic angular dependence of dipolar systems [25]. However, in the case where the quantum number is not conserved $\Delta m \neq 0$, the associated spherical harmonics have a different angular dependence and also take complex values $\propto e^{-i \Delta m \varphi}$. Such spin-orbit process, which appears naturally in the dipole-dipole interactions, is the main building block of recent proposals to observe topological phases via dipolar systems [26].

\subsection{Application: anisotropic dipole-dipole interactions}

Before we present the perturbative regime of the dipole-dipole interactions, we show an elementary example of application regarding the calculation of dipole-dipole matrix elements, which applies for a recent experimental measurement [27]. We consider, for the two Rubidium atoms 1, 2, the coupling between the two-body state $|a b\rangle=$ $\left|59 d_{3 / 2}, m_{j}=3 / 2,59 d_{3 / 2}, m_{j}=3 / 2,\right\rangle$ and $|c d\rangle=\mid 61 p_{1 / 2}, m_{j}=1 / 2,57 f_{5 / 2}, m_{j}=$ $5 / 2+\Delta m\rangle$. The angular dependence of the dipole-dipole matrix element is shown in Fig. 4, for the different processes $\Delta m=0,-1,-2$. In the angular momentum conserving case $\Delta m=0$, we obtain the familiar dipole-dipole potential curve $\propto$ $1-3 \cos ^{2} \theta$, whereas the processes for $\Delta m$ involves another spherical harmonic. Note that in this case the matrix element vanishes at $\theta=0$, as a consequence of the conservation of the angular momentum, along the direction of the atoms $[16,17]$.

\section{Van der Waals regime}

The calculation of the interaction Hamiltonian between two Rydberg atoms requires in general a very large basis $\left|n_{a} X_{a} m_{a}, n_{c} X_{x} m_{c}\right\rangle$ corresponding to all the states coupled by the dipole-dipole interaction within a given energy range. However, at large 


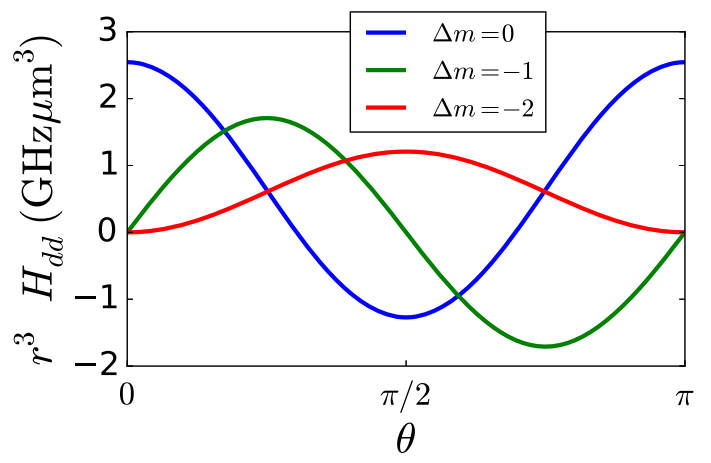

Fig. 4. Angular dependence of the dipole-dipole interaction for different processes $\Delta m=$ $0,-1,-2$, shown respectively in blue, green and red.

distances and away from Förster resonances [3], it is possible to treat these interactions perturbatively, and obtain a reduced van der Waals (vdW) Hamiltonian, governing the interaction in a reduced Hilbert space. At large magnetic field, where the typical interaction strength is smaller than the Zeeman shifts, this reduced Hilbert space corresponds to a single Rydberg pair state and the associated energy can be written as $C_{6} / r^{6}$, where $C_{6}$ is the well-known van der Waals coefficient [3]. Here we are interested in a more general scenario where we take into account the coupling between different Zeeman (aka magnetic) states, and write the vdW Hamiltonian in the corresponding fine-structure basis.

\subsection{Perturbative coupling between fine-structure manifolds}

To do so, we consider two atoms in a Rydberg state, which belongs to the finestructure manifolds $n_{a} X_{a}, n_{b} X_{b}$ (for example $n_{a} s_{1 / 2}, n_{b} s_{1 / 2}$ ). Now we write the part of the dipole-dipole Hamiltonian, which connects the set $n_{a} X_{a}, n_{b} X_{b}$ to another set of manifolds: $n_{c} X_{c}, n_{d} X_{d}$ (for example $n_{c} p_{1 / 2}, n_{d} p_{1 / 2}$ )

$$
\begin{aligned}
h= & \frac{C_{3}\left(n_{a} X_{a} n_{b} X_{b}, n_{c} X_{c} n_{d} X_{d}\right)}{r^{3}} \sum_{m_{c}, m_{d}}\left[\sum _ { m _ { a } , m _ { b } } \left[\mathcal{D}\left(X_{a} m_{a} X_{b} m_{b}, X_{c} m_{c} X_{d} m_{d}\right)\right.\right. \\
& \left.\times\left|n_{a} X_{a} m_{a}, n_{b} X_{b} m_{b}\right\rangle\left\langle n_{c} X_{c} m_{c}, n_{d} X_{d} m_{d}\right|+\text { h.c }\right] \\
& +\delta\left(X_{b} n_{c}, n_{d} X_{d}\right)\left|n_{c} X_{c} m_{c}, n_{d} X_{d} m_{d}\right\rangle\left\langle n_{c} X_{c} m_{c}, n_{d} X_{d} m_{d}\right|
\end{aligned}
$$

where $\delta\left(n_{c} X_{c} n_{d} X_{d}\right)=E_{n_{c} X_{c}}+E_{n_{d} X_{d}}-E_{n_{a} X_{a}}-E_{n_{b} X_{b}}$ is the Förster defect, corresponding to the energy mismatch between the two sets of fine-structure manifolds. Note that we assume for simplicity that the Förster defect is independent of the values of the magnetic quantum numbers $m_{a}, m_{b}, m_{c}, m_{d}$, which is valid at small magnetic fields when the Zeeman shifts are much smaller than the Förster defect obtained at zero magnetic field. At large distances, where the condition $C_{3} / r^{3} \ll \delta$ is satisfied, we can treat in second-order perturbation theory the coupling to the states of the manifold $n_{c} X_{c}, n_{d} X_{d}$ and obtain an effective vdW Hamiltonian, restricted to the Hilbert space of the fine-structure $n_{a} X_{a}, n_{b} X_{b}$ :

$$
h \approx \frac{C_{6}\left(n_{c} X_{c} n_{d} X_{d}\right)}{r^{6}} \mathcal{M}\left(X_{c} X_{d}\right),
$$


Table 1. Channels involved $X_{c}^{(i)} X_{d}^{(i)}$ in the vdW Hamiltonian of two atoms, excited in a $X_{a}, X_{b}$ state, respectively.

\begin{tabular}{c|c|c|c|c|c}
$X_{a} X_{b}$ & $s_{1 / 2} s_{1 / 2}$ & $p_{1 / 2} p_{1 / 2}$ & $p_{3 / 2} p_{3 / 2}$ & $d_{3 / 2} d_{3 / 2}$ & $d_{5 / 2} d_{5 / 2}$ \\
\hline \hline$X_{c}^{(1)} X_{d}^{(1)}$ & $p_{1 / 2} p_{1 / 2}$ & $s_{1 / 2} s_{1 / 2}$ & $s_{1 / 2} s_{1 / 2}$ & $p_{1 / 2} p_{1 / 2}$ & $p_{3 / 2} p_{3 / 2}$ \\
$X_{c}^{(2)} X_{d}^{(2)}$ & $p_{3 / 2} p_{1 / 2}$ & $d_{3 / 2} s_{1 / 2}$ & $s_{1 / 2} d_{3 / 2}$ & $p_{1 / 2} p_{3 / 2}$ & $p_{3 / 2} f_{5 / 2}$ \\
$X_{c}^{(3)} X_{d}^{(3)}$ & $p_{1 / 2} p_{3 / 2}$ & $s_{1 / 2} d_{3 / 2}$ & $d_{3 / 2} s_{1 / 2}$ & $p_{3 / 2} p_{1 / 2}$ & $f_{5 / 2} p_{3 / 2}$ \\
$X_{c}^{(4)} X_{d}^{(4)}$ & $p_{3 / 2} p_{3 / 2}$ & $d_{3 / 2} d_{3 / 2}$ & $s_{1 / 2} d_{5 / 2}$ & $p_{1 / 2} f_{5 / 2}$ & $p_{3 / 2} f_{7 / 2}$ \\
$X_{c}^{(5)} X_{d}^{(5)}$ & & & $d_{5 / 2} s_{1 / 2}$ & $f_{5 / 2} p_{1 / 2}$ & $f_{7 / 2} p_{3 / 2}$ \\
$X_{c}^{(6)} X_{d}^{(6)}$ & & & $d_{3 / 2} d_{3 / 2}$ & $p_{3 / 2} p_{3 / 2}$ & $f_{5 / 2} f_{5 / 2}$ \\
$X_{c}^{(7)} X_{d}^{(7)}$ & & & $d_{3 / 2} d_{5 / 2}$ & $p_{3 / 2} f_{5 / 2}$ & $f_{5 / 2} f_{7 / 2}$ \\
$X_{c}^{(8)} X_{d}^{(8)}$ & & & $d_{5 / 2} d_{3 / 2}$ & $f_{5 / 2} p_{3 / 2}$ & $f_{7 / 2} f_{5 / 2}$ \\
$X_{c}^{(9)} X_{d}^{(9)}$ & & & $d_{5 / 2} d_{5 / 2}$ & $f_{5 / 2} f_{5 / 2}$ & $f_{7 / 2} f_{7 / 2}$
\end{tabular}

where the vdW coefficient is

$$
C_{6}\left(n_{c} X_{c} n_{d} X_{d}\right)=-\frac{C_{3}^{2}\left(n_{a} X_{a} n_{b} X_{b}, n_{c} X_{c} n_{d} X_{d}\right)}{\delta\left(n_{c} X_{c} n_{d} X_{d}\right)}
$$

and the operator $\mathcal{M}\left(X_{c} X_{d}\right)$ can be written in the fine-structure basis $\left\{\left|n_{a} X_{a} m_{a}, n_{b} X_{b} m_{b}\right\rangle\right\}$ :

$$
\begin{aligned}
& \left\langle n_{a} X_{a} m_{a}, n_{b} X_{b} m_{b}\left|\mathcal{M}\left(X_{c} X_{d}\right)\right| n_{a} X_{a} m_{a}^{\prime}, n_{b} X_{b} m_{b}^{\prime}\right\rangle \\
& =\sum_{m_{c}, m_{d}} \mathcal{D}\left(X_{a} m_{a} X_{b} m_{b}, X_{c} m_{c} X_{d} m_{d}\right) \mathcal{D}^{*}\left(X_{a} m_{a}^{\prime} X_{b} m_{b}^{\prime}, X_{c} m_{c} X_{d} m_{d}\right) .
\end{aligned}
$$

The Hamiltonian (12) is thus defined in a Hilbert space of reduced dimension $\left(2 j_{a}+1\right)\left(2 j_{b}+1\right)$, compared to the original dipole-dipole Hamiltonian Eq. (11). We now repeat the same procedure for all dipole-dipole coupled $\left(n_{c} X_{c}, n_{d} X_{d}\right)$ sets of fine-structure manifolds.

\subsection{Van der Waals Hamiltonians}

We can obtain the vdW Hamiltonian in a compact form by noticing that the angular $\mathcal{M}$ matrices do not depend on the first quantum numbers $n_{c}, n_{d}$, allowing to group the different contributions in terms of channels $i=1,2, .$. associated with the quantum numbers $X_{c}^{(i)} X_{d}^{(i)}[15,17,21]$ and listed in Table 1 . The final form of the vdW Hamiltonian reads

$$
h=\sum_{i} \frac{C_{6}^{(i)}}{r^{6}} \mathcal{M}_{i}
$$

with

$$
C_{6}^{(i)}=\sum_{n_{c}, n_{d}} C_{6}\left(n_{c} X_{c}^{(i)} n_{d} X_{d}^{(i)}\right)
$$

and $\mathcal{M}_{i} \equiv \mathcal{M}\left(X_{c}^{(i)} X_{d}^{(i)}\right)$. The $C_{6}$ coefficients are calculated from Eq. (16) using the values of the $C_{3}$ coefficients and of the Rydberg energies. We show in Fig. 5 their values in the usual situation where the state of both atoms belongs to the same manifold: $n_{b}=n_{a}, X_{b}=X_{a}$. Note that some values of the $C_{6}^{(i)}$ coefficients are identical, for instance for $i=2,3$ in the first two columns. In the case of $p_{3 / 2}$ and $d_{3 / 2}$ states, the divergences of the $C_{6}$ coefficients put into evidence the presence of Förster resonances where the Förster defect $\delta$ is nearly zero. 

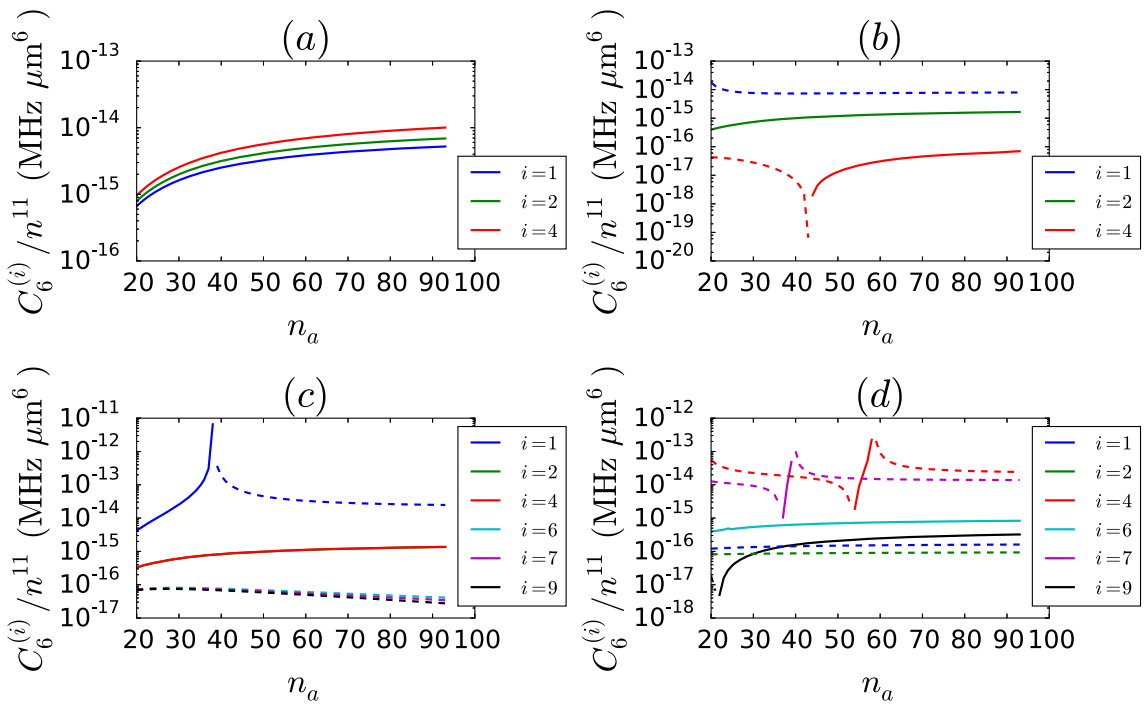

Fig. 5. Van der Waals coefficients $C_{6}^{(i)}$ of the channels [Table 1], associated with the Rubidium Rydberg states $s_{1 / 2}, p_{1 / 2}, p_{3 / 2}, d_{3 / 2}$, shown respectively in panels (a), (b), (c) and (d). The values are shown as a solid line for positive values, as a dashed line otherwise.

\subsubsection{The familiar $s_{1 / 2}$ states}

In the case of $X_{a}=X_{b}=s_{1 / 2}$ states, the vdW Hamiltonian can be written in the form [19]:

$$
\begin{aligned}
h= & \frac{2}{27 r^{6}}\left(\frac{1}{3}\left[2 C_{6}^{(1)}+14 C_{6}^{(2)}+11 C_{6}^{(4)}\right] \mathbb{I}\right. \\
& \left.+\left(-C_{6}^{(1)}+2 C_{6}^{(2)}-C_{6}^{(4)}\right)\left[\begin{array}{cccc}
-\sin ^{2} \theta & -\frac{1}{2} \sin 2 \theta & -\frac{1}{2} \sin 2 \theta & \sin ^{2} \theta \\
-\frac{1}{2} \sin 2 \theta & \sin ^{2} \theta-\frac{2}{3} & \sin ^{2} \theta-\frac{4}{3} & \frac{1}{2} \sin 2 \theta \\
-\frac{1}{2} \sin 2 \theta & \sin ^{2} \theta-\frac{4}{3} & \sin ^{2} \theta-\frac{2}{3} & \frac{1}{2} \sin 2 \theta \\
\sin ^{2} \theta & \frac{1}{2} \sin 2 \theta & \frac{1}{2} \sin 2 \theta & -\sin ^{2} \theta
\end{array}\right]\right),
\end{aligned}
$$

written here in the basis $\left(\left|-\frac{1}{2},-\frac{1}{2}\right\rangle,\left|-\frac{1}{2}, \frac{1}{2}\right\rangle,\left|\frac{1}{2},-\frac{1}{2}\right\rangle,\left|\frac{1}{2}, \frac{1}{2}\right\rangle\right)$ where we use in the following the shorthand notation $\left|m_{a} m_{b}\right\rangle \equiv\left|n_{a} X_{a} m_{j}=m_{a}, n_{b} X_{b} m_{j}=m_{b}\right\rangle$. The matrix $\mathbb{I}$ is the $4 \times 4$ identity matrix. The first term in Eq. (17) describes the isotropic part of the interaction, corresponding to the well-known van-der Waals shift, which is at the heart of the Rydberg blockade phenomenon [4]. The second term represents the contribution of the anisotropic and the state-mixing character of the vdW interactions. The anisotropy refers to the fact that the interaction between two atoms in a given $m_{j}$ state depends on the angle $\theta$ between their relative vector and the direction of the quantization axis. The state-mixing character corresponds to the fact, that in analogy to the dipole-dipole interactions presented in the previous section, the vdW interactions couple different angular momentum states. We emphasize that, in 
(a)

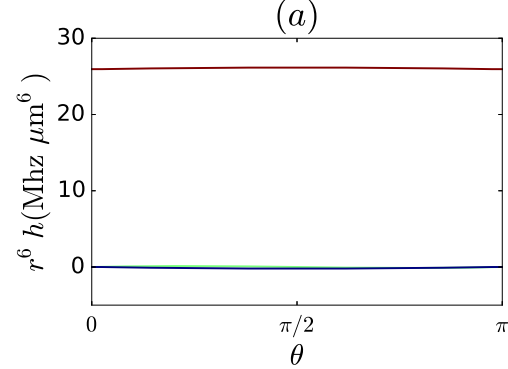

(c)

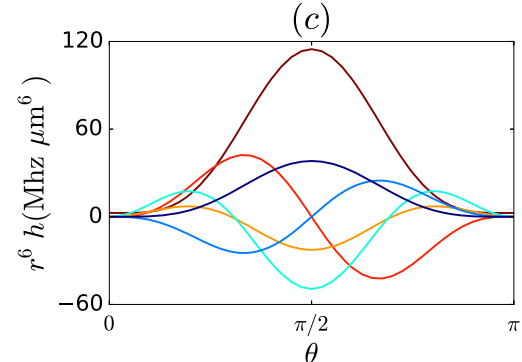

$(e)$

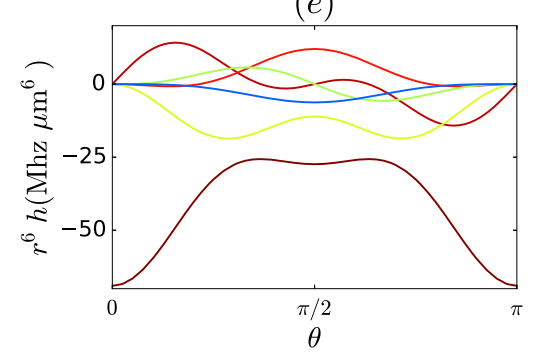

(b)

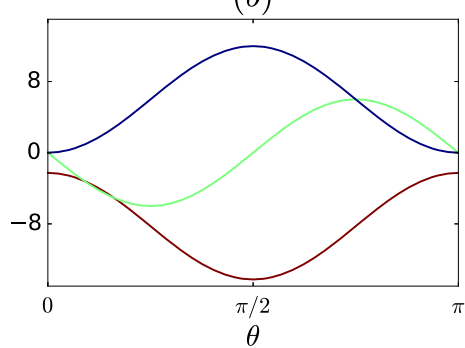

$(d)$
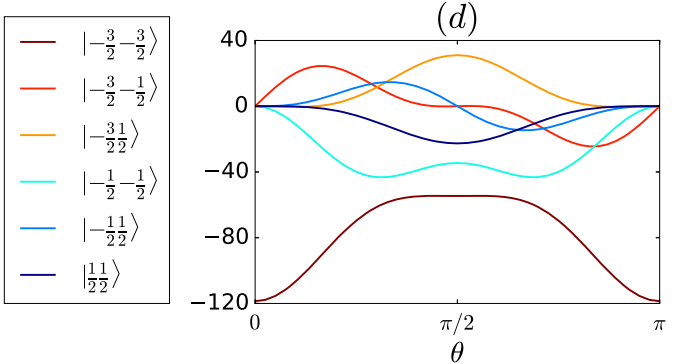

$(f)$
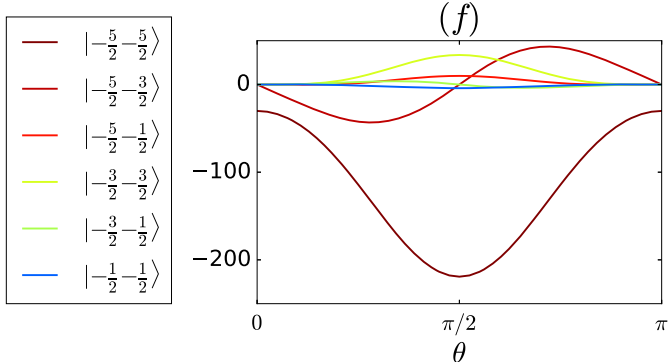

Fig. 6. Examples of matrix elements of the vdW Hamiltonian Eq. (15), for $n=30$ Rubidium atoms and (a) $s_{1 / 2}$, (b) $p_{1 / 2}$, (c) $p_{3 / 2}$, (d) $d_{3 / 2}$, (e) $d_{5 / 2}$ and (f) $f_{5 / 2}$ states. In each panel, we represent the matrix elements $\left\langle m_{a} m_{b}|h| m_{a}^{\prime} m_{b}^{\prime}\right\rangle$, where $m_{a}=m_{b}=-j$ and $m_{a}^{\prime}, m_{b}^{\prime}$ are shown in the legends. The phase $\varphi$ has been set to 0 so that all matrix elements are real.

the case of $s$ states, the existence of the second term is only due to the fine-structure splitting on the neighboring $p$ states, cf. Table 1 . Consequently, this term corresponds to a small deviation compared to the isotropic, diagonal term.

Finally, the matrix elements of the vdW Hamiltonian depend on the angle $\theta$, but not the corresponding eigenvalues $[17,19]$ which are isotropic. However, the presence of a magnetic field along the $z$ direction, breaking the degeneracy between the different angular momentum states, makes the eigenvalues of the total Hamiltonian depend on $\theta$ and gives the anisotropy of the $\mathrm{vdW}$ interactions a physical meaning.

As an illustration, we show in Fig. 6(a) three matrix elements of the vdW Hamiltonian Eq. (17). The diagonal term $\left\langle-\frac{1}{2}-\frac{1}{2}|h|-\frac{1}{2}-\frac{1}{2}\right\rangle$ represented as a blue line, is much larger than the off-diagonal elements, showing that the effect of the statemixing is negligible for Rydberg $s_{1 / 2}$ states. Moreover, the potential curve is almost independent of the value of the angle $\theta$, illustrating the isotropic character of the interactions. 


\subsubsection{Rydberg $p_{1 / 2}$ states}

We now consider the case of $p_{1 / 2}$ states, where the state-mixing and the anisotropy now play an important role. The vdW Hamiltonian can also be written in the form Eq. (17), with the crucial difference that the $C_{6}^{(i)}$ take different values, see Fig. 5 . Consequently, the second term related to the anisotropic and state mixing character of the interactions is no longer negligible compared to the case of $s_{1 / 2}$ states. We show in Fig. 6(b) the same matrix elements as in (a): except at $\theta=0$, where the angular momentum is conserved, the state-mixing processes, represented as green and red lines, are of the same order of magnitude as the diagonal matrix element. Moreover, in contrast to $s_{1 / 2}$ states all three processes have a strong angular dependence.

\subsubsection{Higher angular momentum Rydberg states: $p_{3 / 2}, d_{3 / 2}, d_{5 / 2}, f_{5 / 2}$}

In the case of Rydberg states with higher angular momentum $j=3 / 2,5 / 2, .$. , the vdW Hamiltonian, written in the product state basis $|-j, j\rangle,|-j, j+1\rangle, .$. , involves $(2 j+1)^{2}$ states. Despite the fact that such Rydberg states are associated with a larger Hilbert space, the anisotropic and state-mixing features are very similar compared to the case of $p_{1 / 2}$ states. To conclude this section, we show in Fig. 6(c)-(f) the angular dependence of some matrix elements of the vdW Hamiltonians with the same notations as in the case of $s_{1 / 2}$ and $p_{1 / 2}$ states [(a)-(b)].

\section{Applications}

The goal of this section is to make use of the theoretical toolbox presented in the previous sections to discuss the impact and the possible applications of the statemixing and anisotropic character of the vdW interactions. We first present the results obtained in Ref. [18] where we describe a many-body system excited to a Rydberg $p$ state and interacting via the vdW anisotropic potential. We then present a theory of generalized Rydberg blockade taking into account the effect of state-mixing for mesoscopic Rydberg ensembles [19].

\subsection{Anisotropic interactions for Rydberg crystals}

In a recent work [18], we studied the consequence of such anisotropy on the formation of Rydberg crystals. The system we have in mind is shown in Fig. 7: we consider a set $i=1, . ., N$ of atoms, placed at positions $\mathbf{r}_{i}$, in a two-dimensional square lattice and excited from a ground state level $|\downarrow\rangle$ to a Rydberg level $|\uparrow\rangle$. We are interested in the situation where a strong magnetic field induces a large Zeeman shift between the magnetic levels so that we can ignore state-mixing processes, and reduce the vdW Hamiltonian to a scalar, anisotropic, energy shift. The many-body Hamiltonian can be written as:

$$
H_{\mathrm{C}}=\frac{\hbar}{2} \sum_{i=1}^{N}\left(\Omega \sigma_{x}^{(i)}-\Delta \sigma_{z}^{(i)}\right)+\frac{1}{2} \sum_{i=1}^{N} \sum_{k=1, k \neq i}^{N} \frac{C_{6}\left(\theta_{i, k}\right) P_{i} P_{k}}{\left|\mathbf{r}_{i}-\mathbf{r}_{k}\right|^{6}},
$$

where $\sigma_{x}^{(i)}=|\uparrow\rangle_{i}\langle\downarrow|+| \downarrow\rangle_{i}\left\langle\uparrow\left|, \sigma_{z}^{(i)}=\right| \uparrow\right\rangle_{i}\langle\uparrow|-| \downarrow\rangle_{i}\langle\downarrow|$ refer to the Pauli matrices, $P_{i}=|\uparrow\rangle_{i}\langle\uparrow|$ is the projection on the Rydberg level, $\Omega, \Delta$ denote, respectively the Rabi frequency and the detuning associated with the laser-excitation and $C_{6}\left(\theta_{i, k}\right)$ 
(a)

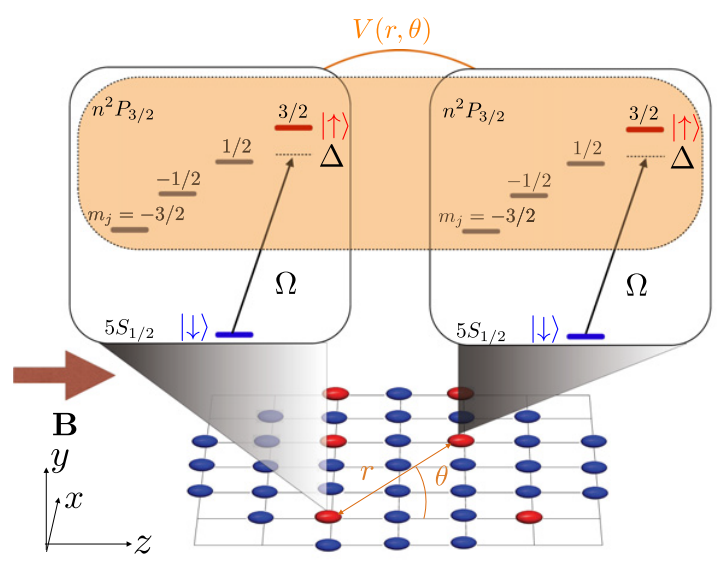

(b)

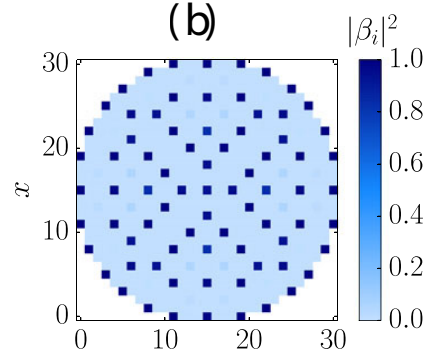

(c)

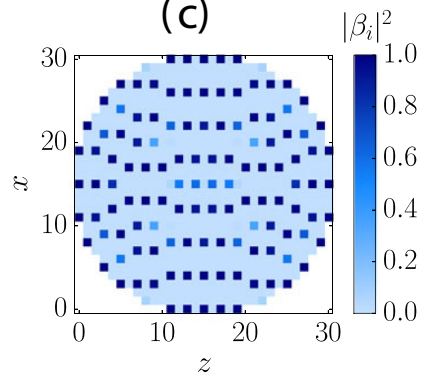

Fig. 7. Anisotropic Rydberg crystal obtained by dissipative state preparation of Rydberg $p_{3 / 2}$ states. The graphics are reproduced from [18].

corresponds to the vdW coefficient associated with the interaction between Rydberg states and defined as ${ }_{i, k}\langle\uparrow \uparrow|h| \uparrow \uparrow\rangle_{i, k}=C_{6}\left(\theta_{i, k}\right) /\left|\mathbf{r}_{i}-\mathbf{r}_{k}\right|^{6}$.

We are interested in the ground-state $|G\rangle$ of this model, corresponding to the zerotemperature phase-diagram. A particularly interesting case is the so-called "classical limit" $\Omega=0$, where the Fock states, or classical configurations, $\left|\prod_{i} s_{i}\right\rangle$, with $s_{i}=\uparrow, \downarrow$, are eigenstates of the Hamiltonian. For $\Delta<0$, the energy is minimal when all spins are polarized $|G\rangle=|\downarrow . . \downarrow\rangle$ (we consider without loss of generality, that the vdW interactions are repulsive). On the other hand, for $\Delta>0$, the energy of the upper state $\uparrow$ becomes larger than the one of the lower state $\downarrow$, thus making the presence of Rydberg excitations energetically favorable. Due to the vdW interactions, these excitations tend to be distant from each other leading to a crystalline pattern $[2,28,29]$.

In an experiment, such Rydberg crystals, which have been observed in the case of isotropic $s_{1 / 2}$ states [30] are prepared by dissipative state preparation: the parameters of the laser $\Omega$ and $\Delta$ are varied in time starting from a known ground-state configuration $\Omega=0, \Delta<0$ to end in the crystalline region $\Omega \neq 0, \Delta>0$. The question addressed in Ref. [18] is the role of the anisotropic vdW interactions in the formation of Rydberg Crystals involving $p$ or $d$ states. To this end, we studied via a time-dependent mean-field approach, which is in particular exact in the classical limit, the preparation of such crystals. As an illustration, we represent in Figs. 7(b)-(c), the ground-state excitation pattern, obtained at the end of the preparation for two different configurations. In the isotropic configuration, corresponding to a magnetic field orientation perpendicular to the plane, the Rydberg excitation pattern is invariant by a rotation of 90 degrees. In the the anisotropic case, where the magnetic field is set along the plane, the crystal is preferentially aligned along the $x$ axis, where the interactions are the weakest. Finally, we studied the role of the quantum fluctuations which depend on the value of the laser Rabi frequency $\Omega$, leading to a progressive melting of the crystalline phase towards a disordered phase. 
(a)

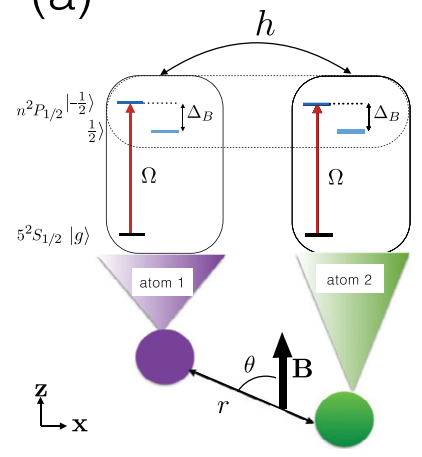

(b)

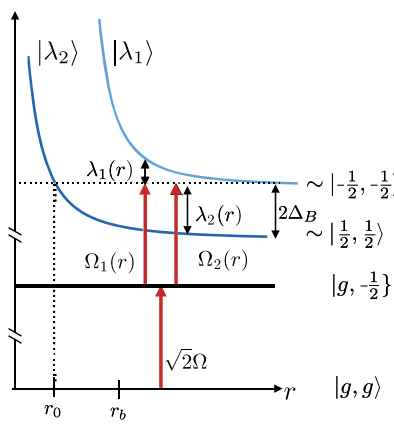

(c)

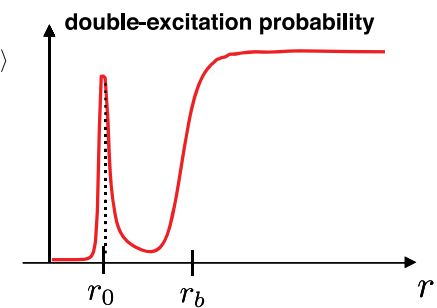

Fig. 8. Generalized Rydberg blockade with state-mixing, represented here for two atoms laser-excited to a Rydberg $p_{1 / 2}$ states. The graphics are reproduced from [19]. (This figure is subject to copyright protection and is not covered by a Creative Commons license.)

\subsection{Anisotropic Rydberg blockade with state-mixing: magic distances}

In the case of weak or moderate magnetic fields, the coupling between different magnetic levels of the fine-structure cannot be ignored. In Ref. [19], we investigate its consequences on the well-know Rydberg blockade phenomenon [4]. To this end, we first consider the situation, shown in Fig. 8, where two atoms are excited via a laser to a Rydberg $p_{1 / 2}$ state and interact via vdW interactions. The corresponding Hamiltonian can be written in the form $H_{\mathrm{M}}=\sum_{i=1,2} H_{A}^{(i)}+h$, see Fig. 8(a), with:

$$
H_{A}^{(i)}=\frac{\hbar \Omega}{2}\left[|g\rangle_{i}\left\langle-\frac{1}{2}|+|-\frac{1}{2}\right\rangle_{i}\langle g|\right]-\hbar \Delta_{B}\left|\frac{1}{2}\right\rangle_{i}\left\langle\frac{1}{2}\right|,
$$

where $g$ is a ground state level, $h$ is given in Eq. (17) and $\Delta_{B}$ represents the Zeeman shift induced by the magnetic field. The limiting case $\theta=\pi / 2$ represents the simple example of state-mixing where the doubly laser-excited state $\left|-\frac{1}{2}-\frac{1}{2}\right\rangle$ is only coupled via the vdW interactions to $\left|\frac{1}{2} \frac{1}{2}\right\rangle$. In this case, considering that initially the two atoms are in the ground state $|g g\rangle$, we can restrict the Hilbert space to four states: $|g g\rangle$, $\left.\mid g-\frac{1}{2}\right\} \equiv 1 \sqrt{2}\left(\left|g-\frac{1}{2}\right\rangle+\left|-\frac{1}{2} g\right\rangle\right)$ and the doubly excited states $\left|-\frac{1}{2}-\frac{1}{2}\right\rangle,\left|\frac{1}{2} \frac{1}{2}\right\rangle$, with the reduced Hamiltonian

$$
h_{\mathrm{M}}=\left[\begin{array}{cccc}
0 & \hbar \Omega / \sqrt{2} & 0 & 0 \\
\hbar \Omega / \sqrt{2} & 0 & \hbar \Omega / \sqrt{2} & 0 \\
0 & \hbar \Omega / \sqrt{2} & u / r^{6} & c / r^{6} \\
0 & 0 & c / r^{6} & u / r^{6}-2 \hbar \Delta_{B} .
\end{array}\right] \text {, }
$$

where $u, c$ are a sum of $C_{6}^{(i)}$ coefficients, representing respectively the energy shift and the coupling between magnetic levels. In the familiar Rydberg blockade picture associated with $s_{1 / 2}$ states, the term $c$ is neglected. Consequently, the doubly excited state $\left|-\frac{1}{2}-\frac{1}{2}\right\rangle$ can only be excited at large distances, where the interaction $u$ is negligible compared to the laser excitation bandwidth $\propto \Omega$ : this is the well-known Rydberg blockade phenomenon. 
The goal of our work consisted in investigating how the mixing term $c$, which is of the same order as $u$ for $p_{1 / 2}$ affects the Rydberg blockade phenomenon. To this end, an insightful representation of the problem is obtained by diagonalizing the Hamiltonian in the doubly-excited manifold $\left\{\left|-\frac{1}{2}-\frac{1}{2}\right\rangle,\left|\frac{1}{2} \frac{1}{2}\right\rangle\right\}$, obtaining two energies $\lambda_{1}(r), \lambda_{2}(r)$. The representation of the Born-Oppenheimer (BO) potentials $\lambda_{1}(r), \lambda_{2}(r)$ as a function of the distance $r$ between the atoms, represented schematically in Fig. 8(b), allows one to know whether the doublyexcited manifold can be excited or not, generalizing thus the Rydberg blockade phenomenon.

A particularly interesting situation corresponds to the case where the Zeeman shift $\Delta_{B}$ competes with the vdW interactions $(u, c)$ : at large distances, one of the $\mathrm{BO}$ energies is, for instance, negative, due to the Zeeman shift, while at short distances, it becomes positive due to the vdW interactions. Consequently, there is a "magic" distance $r=r_{0}$, where this energy is zero, allowing for a resonance excitation of the doubly excited state [see Figs. 8(b)-(c)]. At this particular distance, interactions still play an important role however, as the state $\left|\lambda_{1}\right\rangle$, which is excited, is a linear superposition of the two product states $\left|-\frac{1}{2}-\frac{1}{2}\right\rangle,\left|\frac{1}{2} \frac{1}{2}\right\rangle$. This has interesting consequences at the many-body level: considering $p=1, . ., M$ pairs of atoms separated by the magic distance: due to the superposition character of the magic distance state $\left|\lambda_{1}\right\rangle$, the many-body state $|\Psi\rangle=\otimes_{p}\left|\lambda_{1}\right\rangle_{p}$ is not a zero-energy eigenstate of the vdW Hamiltonian $\sum_{p} h_{p}$. The consequence of this phenomenon, related to the non-additivity properties of the interaction Hamiltonian (see [31] in the context of dipole-dipole interactions), is a blockade mechanism: in a blockade sphere, defined as the region where interactions dominate over the laser-excitation, it is not possible to excite more than one magic-distance state $\left|\lambda_{1}\right\rangle$.

Finally, the analytical understanding we developed in the context of $p_{1 / 2}$ can be extended to the case of higher angular momentum states: $p_{3 / 2}, d_{3 / 2}$ states in particular. As an illustration, we applied our approach to give a theoretical understanding of recent experimental results [32], showing the angular dependence of the vdW interactions between Rydberg $d_{3 / 2}$ states, in an situation where the magnitude of the state-mixing processes can be neglected compared to the direct, diagonal, interaction.

\section{Conclusion}

In summary, we have presented the complete theoretical toolbox, necessary to calculate the dipole-dipole and van der Waals interactions between Rydberg states. We put in particular into evidence the anisotropic character of the vdW interactions, and the presence of state-mixing processes, i.e coupling terms between different magnetic levels. Finally, we summarized recent applications of this theoretical framework to the description of exotic many-body phases [18] and unconventional Rydberg blockade [19], both of them being relevant for the interpretation of on-going and future experiments. The possibilities given by these rich and complex properties of the Rydberg interactions are manifold: observation of topological states of matter [26], implementation of exotic spin models [33,34], new types of interactions between Rydberg polaritons [35], or the implementation of chiral spin-wave guides, where Rydberg excitations propagate along a chain of atoms along a preferential direction [36].

BV acknowledges funding from the ERC-Synergy Grant UQUAM and the Marie Curie Initial Training Network COHERENCE. Work in Innsbruck is also supported by the European Research Council (ERC) programs SIQS, RYSQ and the Spezial- Forschungsbereich 
(SFB) FoQuS, Austrian Science Fund (FWF) Project No. F4016-N23. The author thanks M. Punk, A. Glaetzle and P. Zoller who participate to the works $[18,19]$, whose results are presented in the end of this article and is also very thankful to A. Glaetzle for introducing him the physics of interacting Rydberg atoms, and C. Dlaska for useful comments on the manuscript.

\section{References}

1. T.F. Gallagher, Rydberg Atoms, Cambridge Monographs on Atomic, Molecular and Chemical Physics (Cambridge University Press, 2005)

2. R. Löw, H. Weimer, J. Nipper, J.B. Balewski, B. Butscher, H. Büchler, T. Pfau, J. Phys. B: At. Mol. Opt. Phys. 45, 113001 (2012)

3. M. Saffman, T.G. Walker, K. Mølmer, Rev. Mod. Phys. 82, 2313 (2010)

4. D. Jaksch, J.I. Cirac, P. Zoller, S.L. Rolston, R. Côté, M.D. Lukin, Phys. Rev. Lett. 85, $2208(2000)$

5. M.D. Lukin, M. Fleischhauer, R. Cote, L.M. Duan, D. Jaksch, J.I. Cirac, P. Zoller, Phys. Rev. Lett. 87, 37901 (2001)

6. A. Gaëtan, Y. Miroshnychenko, T. Wilk, A. Chotia, M. Viteau, D. Comparat, P. Pillet, A. Browaeys, P. Grangier, Nat. Phys. 5, 115 (2009)

7. E. Urban, T.A. Johnson, T. Henage, L. Isenhower, D.D. Yavuz, T.G. Walker, M. Saffman, Nat. Phys. 5, 110 (2009)

8. D. Tong, S.M. Farooqi, J. Stanojevic, S. Krishnan, Y.P. Zhang, R. Côté, E.E. Eyler, P.L. Gould, Phys. Rev. Lett. 93, 063001 (2004)

9. K. Singer, M. Reetz-Lamour, T. Amthor, L.G. Marcassa, M. Weidemüller, Phys. Rev. Lett. 93, 163001 (2004)

10. P. Cheinet, S. Trotzky, M. Feld, U. Schnorrberger, M. Moreno-Cardoner, S. Fölling, I. Bloch, Phys. Rev. Lett. 101, 090404 (2008)

11. P. Schauß, M. Cheneau, M. Endres, T. Fukuhara, S. Hild, A. Omran, T. Pohl, C. Gross, S. Kuhr, I. Bloch, Nature 491, 87 (2012)

12. N. Malossi, M.M. Valado, S. Scotto, P. Huillery, P. Pillet, D. Ciampini, E. Arimondo, O. Morsch, Phys. Rev. Lett. 113, 023006 (2014)

13. H. Schempp, G. Günter, M. Robert-de Saint-Vincent, C.S. Hofmann, D. Breyel, A. Komnik, D.W. Schönleber, M. Gärttner, J. Evers, S. Whitlock, M. Weidemüller, Phys. Rev. Lett. 112, 013002 (2014)

14. J. Zeiher, P. Schauß, S. Hild, T. Macrì, I. Bloch, C. Gross, Phys. Rev. X 5, 031015 (2015)

15. A. Reinhard, T. Liebisch, B. Knuffman, G. Raithel, Phys. Rev. A 75, 032712 (2007)

16. K. Singer, J. Stanojevic, M. Weidemüller, R. Côté, J. Phys. B: At. Mol. Opt. Phys. 38, S295 (2005)

17. T. Walker M. Saffman, Phys. Rev. A 77, 032723 (2008)

18. B. Vermersch, M. Punk, A.W. Glaetzle, C. Gross, P. Zoller, New J. Phys. 17, 19 (2015)

19. B. Vermersch, A.W. Glaetzle, P. Zoller, Phys. Rev. A 91, 023411 (2015)

20. M. Marinescu, H. Sadeghpour, A. Dalgarno, Phys. Rev. A 49, 982 (1994)

21. A.W. Glaetzle, M. Dalmonte, R. Nath, I. Rousochatzakis, R. Moessner, P. Zoller, Quantum Spin Ice and dimer models with Rydberg atoms 1, 26 (2014)

22. W. Li, I. Mourachko, M. Noel, T. Gallagher, Phys. Rev. A 67, 052502 (2003)

23. J. Han, Y. Jamil, D. Norum, P. Tanner, T. Gallagher, Phys. Rev. A 74, 054502 (2006)

24. J.D. Pritchard, Cooperative Optical Non-linearity in a blockaded Rydberg Ensemble, Ph.D. thesis, Durham University, 2011, p. 210

25. M.A. Baranov, M. Dalmonte, G. Pupillo, P. Zoller, Chem. Rev. 112, 5012 (2012)

26. N.Y. Yao, C.R. Laumann, A.V. Gorshkov, S.D. Bennett, E. Demler, P. Zoller, M.D. Lukin, Phys. Rev. Lett. 109, 266804 (2012)

27. S. Ravets, H. Labuhn, D. Barredo, T. Lahaye, A. Browaeys, Phys. Rev. A 92, 020701(R) (2015) 
28. H. Weimer, H.P. Büchler, Phys. Rev. Lett. 105, 230403 (2010)

29. E. Sela, M. Punk, M. Garst, Phys. Rev. B 84, 085434 (2011)

30. P. Schauß, J. Zeiher, T. Fukuhara, S. Hild, M. Cheneau, T. Macrì, T. Pohl, I. Bloch, C. Gross, Science 347, 1455 (2015)

31. T. Pohl, P. Berman, Phys. Rev. Lett. 102, 013004 (2009)

32. D. Barredo, S. Ravets, H. Labuhn, L. Béguin, A. Vernier, F. Nogrette, T. Lahaye, A. Browaeys, Phys. Rev. Lett. 112, 183002 (2014)

33. A.W. Glaetzle, M. Dalmonte, R. Nath, C. Gross, I. Bloch, P. Zoller, Phys. Rev. Lett. 114, 1 (2015)

34. R.M.W. van Bijnen, T. Pohl, Phys. Rev. Lett. 114, 243002 (2015)

35. C. Tresp, P. Bienias, S. Weber, H. Gorniaczyk, I. Mirgorodskiy, H.P. Büchler, S. Hofferberth, Phys. Rev. Lett. 115, 083602 (2015)

36. B. Vermersch, T. Ramos, P. Hauke, P. Zoller, Phys. Rev. A 93, 063830 (2016)

Open Access This is an Open Access article distributed under the terms of the Creative Commons Attribution License (http://creativecommons.org/licenses/by/4.0), which permits unrestricted use, distribution, and reproduction in any medium, provided the original work is properly cited. 